\title{
Modeling and Comparison of Dissolution Profiles of Diltiazem Modified-Release Formulations
}

\author{
D. Samaha, R. Shehayeb, and S. Kyriacos ${ }^{1}$ \\ Department of Pharmaceutical Sciences, School of Pharmacy, Lebanese American University, \\ P.O. Box 36, Byblos, Lebanon
}

\begin{abstract}
Generic drugs offer a cost-effective alternative to brand-name products. However, the main concern with modified-release formulations is the substitution of one product for another. Accordingly, the first objective of this study was to assess the interchangeability of the available diltiazem extended-release (ER) products on the basis of their in vitro dissolution characteristics using USP Apparatus 2 and 3. The second objective was to compare dissolution profiles in simulated fasted and fed states and determine whether there is a change in the mechanism of drug release. Dissolution profiles characterized using Apparatus 2 or 3 under fasted conditions were similar. However, Apparatus 3 testing provided a more discriminating and comprehensive evaluation of the drug release performance of ER products. Testing using Apparatus 3 in the fed state highlighted some difference in dissolution profiles, suggesting a food effect on drug release. This implies that, depending on the targeted concentration, a patient should be instructed whether to take the medication in fasted or fed state. The study therefore shows the necessity of testing the products in both fasted and fed states to determine their similarity and therapeutic interchangeability. Apparatus 3 testing was also more accurate in determining the release mechanism than the Apparatus 2 method. No change in the mechanism of drug release between fasted and fed state was observed.
\end{abstract}

\section{INTRODUCTION}

( iltiazem is an orally administered, nondihydropyridine calcium-channel blocker that is used for the treatment of hypertension and atrial fibrillation. According to the Biopharmaceutics Classification System (BCS), diltiazem is a Class I substance $(1,2)$, meaning that it is highly soluble and highly permeable.

The drug is completely absorbed throughout the intestinal tract, but it is extensively metabolized in the liver by deacetylation, resulting in incomplete bioavailability (about 35-40\%). Due to its very short half-life of 3.06-6.6 h, it may be necessary to administer immediaterelease formulations up to four times per day, especially in the case of illnesses that require continuous and constant control such as hypertension and angina pectoris (3). Immediate-release dosage forms are also associated with fluctuations in plasma concentrations. To optimize therapy and patient compliance, several extended-release (ER), once- or twice-a-day formulations were developed. Three brands, which are single- or multiple-unit coated tablets or capsules, are available in the Lebanese market.

Generics offer a cost-effective alternative to brand-name products. However, the main concern with modified-release formulations is product substitution. Because the rate or extent of release could differ from one product to another, the patient may be placed at unnecessary risk. Accordingly, the first objective of the

${ }^{1}$ Corresponding author. study was to assess the interchangeability of the available diltiazem extended-release products on the basis of in vitro dissolution characteristics using different apparatus and dissolution media. The second objective was to compare dissolution profiles in simulated fasted and fed states and determine whether there is a change in the mechanism of drug release.

Regulatory developments have introduced the possibility of substituting dissolution tests for clinical studies for some immediate-release formulations. Similar regulations would also be useful for ER formulations. The challenge is to develop dissolution tests that better predict the in vivo performance of drug products (4). USP test methods for diltiazem ER formulations prescribe the use of Apparatus 1 and 2 with simple dissolution media such as water, $0.1 \mathrm{~N} \mathrm{HCl}$, or USP simulated intestinal fluid (SIF). Tests are classified by the frequency of drug administration (once vs. twice daily). These tests, however, do not comprehensively reflect conditions to which a dosage form moving through the human Gl tract will be exposed and therefore cannot be used to predict drug release during the course of Gl passage. To better mimic in vivo conditions, dissolution profiles were established using USP Apparatus 3 with appropriate media to simulate $\mathrm{pH}$ conditions along the Gl tract. Biorelevant gastrointestinal media that simulate the fasted and fed states were developed to predict the in vivo performance of the drug $(5,6)$. Biorelevant in vitro dissolution testing is useful for qualitative forecasting of formulation and food effects on the dissolution and availability of orally 
administered dugs. It has been observed that biorelevant media can provide a more accurate simulation of pharmacokinetic profiles than simulated gastric fluid or simulated intestinal fluid (4, 7-10).

\section{MATERIALS AND METHODS Materials}

Three products were purchased commercially and analyzed:Adizem-XL 120-mg capsules (Napp-mann bard, United Kingdom), Zaldem CR 120-mg capsules (Algorithm, Lebanon), and Bi-tildiem 120-mg tablets (Sanofi-Synthelabo, France). Zaldem CR and Adizem XL are dosed as once daily, whereas Bi-tildiem is dosed as twice daily. All formulations are found as diltiazem hydrochloride. Table 1 summarizes the characteristics of the three products. Because the formulations differ, different release patterns were expected.

Diltiazem $\mathrm{HCl}$ standard substance was purchased from Sigma-Aldrich GmbH (Steinheim,Germany). All reagents used were of analytical grade. Ensure (Abbott

Laboratories) was purchased from the Lebanese market.

The dissolution profiles of twelve tablets were investigated using USP Apparatus 1 or 2 (Pharma Test PTWS, Hainburg, Germany) and Apparatus 3, a reciprocating-cylinder apparatus (BIO-DIS RRT9, Caleva Ltd, Dorset, England).

Reference standard solutions were prepared and analyzed using the same method as for the sample solutions. Drug concentrations were calculated from a linear calibration curve constructed using the standard substance. The linearity of the calibration function was confirmed at four concentrations in the working range.

\section{Experiments with USP Apparatus 1 and 2}

To characterize drug release at the primary site of systemic drug absorption, the small intestine, dissolution testing was performed in Simulated Intestinal Fluid USP (sine pancreatin) (SIFsp) adjusted to a pH of $6.8 \pm 0.1$ with $0.1 \mathrm{M}$ sodium hydroxide or $0.1 \mathrm{M}$ phosphoric acid. The dissolution profiles of the tablets were investigated using Apparatus 1 or 2 at $75 \mathrm{rpm}$. Dissolution media consisted of $900 \mathrm{~mL}$ of SIFsp. Samples ( $5 \mathrm{~mL}$ ) were withdrawn at 0,30, $60,75,90,120,180,960,1320$, and 1440 min using an automatic pipet. A UV spectrophotometer (Jenway, Essex,

Table 1. Characteristics of Drug Products

\begin{tabular}{llll}
\hline $\begin{array}{l}\text { Drug } \\
\text { Product }\end{array}$ & Dosage Form & $\begin{array}{c}\text { Controlled Release } \\
\text { Excipient }\end{array}$ & Release pH \\
\hline Adizem-XL & $\begin{array}{l}\text { Coated } \\
\text { microgranules }\end{array}$ & Ethylcellulose & $\begin{array}{l}\mathrm{pH} \text {-independent } \\
\text { release }\end{array}$ \\
\hline Zaldem CR & $\begin{array}{l}\text { Coated } \\
\text { microgranules }\end{array}$ & Ethylcellulose & $\begin{array}{l}\mathrm{pH} \text {-independent } \\
\text { release }\end{array}$ \\
\hline Bi-Tildiem & $\begin{array}{l}\text { Bi-layer coated } \\
\text { tablets }\end{array}$ & Castor oil & Insoluble \\
\hline
\end{tabular}

Dissolution Technologies | MAY 2009
England) set at $237 \mathrm{~nm}$ was used to analyze the samples for dissolved drug. Dilution was done when necessary. The percentage of drug dissolved at each sampling time point was calculated.

\section{Experiments with USP Apparatus 3}

To simulate passage through the stomach and the small intestine, drug products were additionally tested with a $\mathrm{pH}$-gradient method. Biorelevant gastrointestinal media that simulate the fasted and fed states were used to mimic in vivo conditions and to examine the dissolution characteristics of the three products. Residence times were chosen to represent typical GI transit of solid dosage forms. The $\mathrm{pH}$ and the bile salt (sodium taurocholate) concentration were adjusted to mimic physiological conditions and the active reabsorption of bile salts from the ileum as described by Klein et al. (11) and shown in Table 2.

\section{Preparation of Biorelevant Dissolution Media} Fasted State Simulated Gastric Fluid (FaSSGF)

$\mathrm{SGF}_{\text {Triton }}(\mathrm{pH}$ 1.8) was used to reproduce gastric conditions. Two grams of $\mathrm{NaCl}, 1 \mathrm{~g}$ of Triton $\mathrm{X} 100$, and $3 \mathrm{~g}$ of concentrated $\mathrm{HCl}$ were dissolved with deionized water to make a total volume of $1 \mathrm{~L}$ (12).

\section{Fasted State Simulated Intestinal Fluid (FaSSIF)}

Blank FaSSIF was prepared by dissolving $1.74 \mathrm{~g}$ of $\mathrm{NaOH}$ (pellets), $17.19 \mathrm{~g}$ of anhydrous $\mathrm{NaH}_{2} \mathrm{PO}_{4}$, and $30.93 \mathrm{~g}$ of $\mathrm{NaCl}$ in $5 \mathrm{~L}$ of purified water. The $\mathrm{pH}$ was adjusted to exactly 6.5 using $1 \mathrm{~N} \mathrm{NaOH}$ or $1 \mathrm{~N} \mathrm{HCl}$.

FaSSIF was prepared by dissolving $3.3 \mathrm{~g}$ of sodium taurocholate in $500 \mathrm{~mL}$ of blank FaSSIF and adding 11.8 $\mathrm{mL}$ of a solution of $100 \mathrm{mg} / \mathrm{mL}$ lecithin in methylene chloride, which formed an emulsion. The methylene chloride was eliminated under vacuum for $15 \mathrm{~min}$. The solution was then filtered. This resulted in a clear, micellar solution having no perceptible odor of methylene chloride. After cooling to room temperature, the volume was adjusted to $2 \mathrm{~L}$ with blank FaSSIF.

\section{Table 2. Biorelevant Dissolution Media}

\begin{tabular}{llclc}
\hline & \multicolumn{2}{c}{ Fasted State } & \multicolumn{2}{c}{ Fed State } \\
\cline { 2 - 5 } GI Region & \multicolumn{1}{c}{ Medium } & $\begin{array}{c}\text { Time } \\
(\mathbf{m i n})\end{array}$ & \multicolumn{1}{c}{ Medium } & $\begin{array}{c}\text { Time } \\
\text { (min) }\end{array}$ \\
\hline Stomach & FaSSGF 1.8 & 60 & Ensure & 120 \\
\hline Upper jejunum & FaSSIF 6.5 & 15 & FeSSIF 5.0 & 45 \\
\hline Lower jejunum & FaSSIF 6.8 & 15 & FeSSIF 6.5 & 45 \\
\hline Upper ileum & FaSSIF 7.2** & 30 & FeSSIF 6.5 & 45 \\
\hline Lower ileum & Blank FaSSIF 7.5 & 120 & Blank FaSSIF 7.5 & 45 \\
\hline Proximal colon & Blank FaSSIF 6.5 & 720 & Blank FaSSIF 6.5 & 840 \\
\hline
\end{tabular}

**Halved bile salts. 
To simulate the upper ileum, $1.55 \mathrm{~g}$ of sodium taurocholate was used to constitute halved bile salts (11).

\section{Fed State Simulated Gastric Fluid (FeSGf)}

Although Ensure Plus is very similar to a standard meal in terms of content and physiochemical properties $(13,14)$, Ensure Vanilla Flavor, which is the only Ensure product found in developing countries, was used to simulate a fed stomach. The medium was prepared by dispersing $53.4 \mathrm{~g}$ of Ensure powder in $230 \mathrm{~mL}$ of distilled water (15). The resulting solution was white and viscous. Therefore, to analyze the samples for diltiazem content by UV spectrophotometry, acetonitrile was added to the sampled solution (1:10), and the mixture was centrifuged at $15,000 \mathrm{G}$ for $15 \mathrm{~min}$ at $37^{\circ} \mathrm{C}$, then filtered.

\section{Fed State Simulated Intestinal Fluid (FeSSIF)}

Blank FeSSIF was prepared by dissolving $20.2 \mathrm{~g}$ of $\mathrm{NaOH}$ (pellets), $43.25 \mathrm{~g}$ of glacial acetic acid, and $59.37 \mathrm{~g}$ of $\mathrm{NaCl}$ in $5 \mathrm{~L}$ of purified water. The $\mathrm{pH}$ was adjusted to exactly 5.0 using $1 \mathrm{~N} \mathrm{NaOH}$ or $1 \mathrm{~N} \mathrm{HCl}$.

FeSSIF was prepared by dissolving $16.5 \mathrm{~g}$ of sodium taurocholate in $500 \mathrm{~mL}$ of blank FeSSIF; $59.08 \mathrm{~mL}$ of a solution containing $100 \mathrm{mg} / \mathrm{mL}$ lecithin in methylene chloride was added, forming an emulsion. The methylene chloride was eliminated under vacuum. This resulted in a clear to slightly hazy, micellar solution having no perceptible odor of methylene chloride. After cooling to room temperature, the volume was adjusted to $2 \mathrm{~L}$ with blank FeSSIF (6).

Instruments parameters were selected based on the data presented by Rohrs et al. (16). Both top and bottom of the glass cylinder were fitted with $420-\mu \mathrm{m}$ mesh screen, which is an intermediate value. A volume of $250 \mathrm{~mL}$ of test medium and a standard dip rate of $10 \mathrm{dpm}$ were used in all experiments. Samples $(5 \mathrm{~mL})$ were withdrawn using an automatic pipet at 0,30,60,75,90,120,180,240, and 960 min for the fasted state and at $0,60,120,165,210,255,300$, and 1140 min for the fed state.

Before analysis by UV spectrophotometer, sample solutions were centrifuged for $5 \mathrm{~min}$ at 10000 rotations/ min at $37^{\circ} \mathrm{C}$ and then filtered using a syringe-driven filter unit. Dilution was done when necessary. To analyze drug content in Ensure samples, acetonitrile was used to precipitate the proteins; samples were then centrifuged and filtered.

The similarity factor $\left(f_{2}\right)$ was used to assess the similarity between two dissolution profiles. The similarity factor is defined by FDA and EMEA (17) as

$$
f_{2}=50 \times \log \left\{\left[1+(1 / n) \sum_{j=1}^{n}\left|R_{j}-T_{j}\right|^{2}\right]^{-0.5} \times 100\right\}
$$

where $n$ is the number of time points and $R_{j}$ and $T_{j}$ are the percent dissolved of the reference and test products, respectively, at each time point $j$.
Two dissolution profiles are considered similar when the $f_{2}$ value is between 50 and 100 . To prove similarity, dissolution profiles were compared among one another by $f_{2}$ calculation using dissolution time points from $30 \mathrm{~min}$ to a time corresponding to FDA criteria (i.e., not more than one measurement after $85 \%$ dissolution of the product).

\section{Kinetic Modeling of Dissolution Profiles}

The drug-release mechanism for all the brands in the different media (simulated IF using Apparatus 1 or 2, media simulating the fasted state, and media simulating the fed state) was fitted to zero-order, first-order, Weibull, Higuchi, and Korsmeyer-Peppas models (Table 6).

The results of $F$-statistics were used for determining the most appropriate model. The model with the smallest $F$ value was used for describing the mechanism of drug release.

\section{RESULTS AND DISCUSSION \\ Dissolution Profiles Using Apparatus 2}

The dissolution profiles were first investigated using Apparatus 2. The results are shown in Figure 1. Similarity of dissolution profiles was confirmed by the $f_{2}$ results for Adizem-XL and Zaldem CR, shown in Table 3. The rate of drug release appeared to be higher for Bi-Tildiem. The calculated $f_{2}$ values of about 45 indicate borderline similarity with the other products. Note that in all cases, there was a low variability among brands, with standard deviations less than $5 \%$.

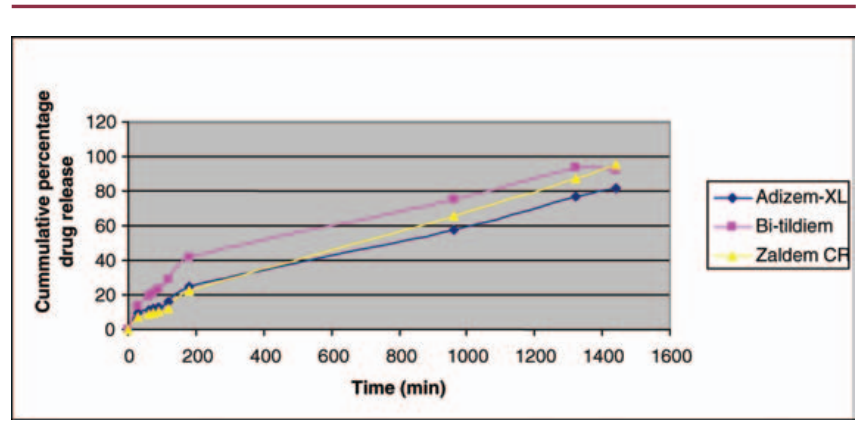

Figure 1.Dissolution profile in simulated IF using USP Apparatus 2. Arithmetic means $(n=12)$ are shown.

Table 3. Similarity Factors for Dissolution Profiles of the Products in Simulated IF

\begin{tabular}{lcc}
\hline Brand & Zaldem CR & Bi-tildiem \\
\hline Adizem-XL & 88.78 & 44.75 \\
\hline Bi-tildiem & 45.39 & - \\
\hline
\end{tabular}

Dissolution Technologies I MAY 2009 


\section{Dissolution Profiles Using Apparatus 3}

Fasted State

The dissolution profiles were also investigated using Apparatus 3 in fasted-state conditions. The results are shown in Figure 2; the dissolution profiles are virtually identical. This is confirmed by the $f_{2}$ results in Table 4 . Therefore, the different ER products are interchangeable when administered in the fasted state.

\section{Fed State}

The dissolution profiles were investigated using Apparatus 3 in fed-state conditions. The results, shown in Figure 3, clearly reflect similarity only between two brands. This is confirmed by the $f_{2}$ results in Table 5 , which show that Bi-Tildiem is significantly different from the other brands.

\section{Fasted State Comparison with Fed State}

To study the effect of food on each formulation, dissolution profiles in fasted and fed conditions were

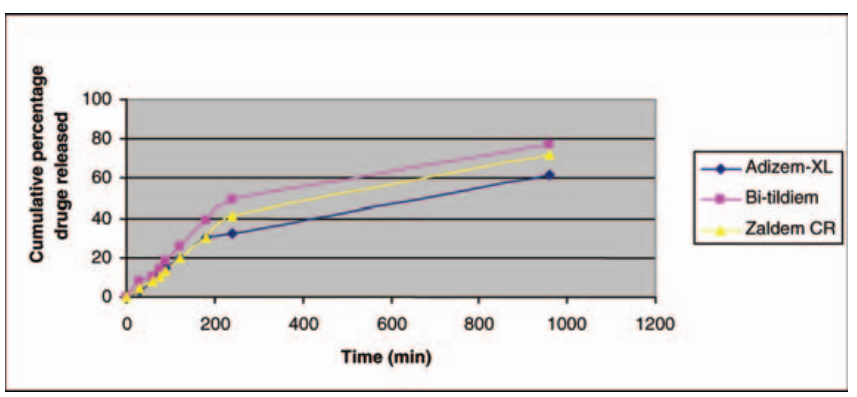

Figure 2. Dissolution profile in fasted-state conditions using USP Apparatus 3.

\section{Table 4. Similarity Factors for Dissolution Profiles of the Products in Fasted State}

\begin{tabular}{lcc}
\hline Brand & Zaldem CR & Bi-tildiem \\
\hline Adizem-XL & 65.77 & 51.37 \\
\hline Bi-tildiem & 61.35 & - \\
\hline
\end{tabular}

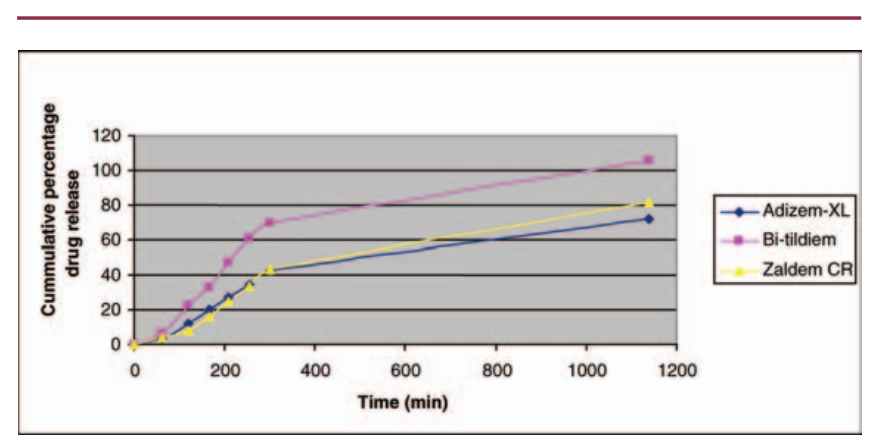

Figure 3. Dissolution profile in fed-state conditions using USP Apparatus 3.
Table 5. Similarity Factors for Dissolution Profiles of the Products in Fed State

\begin{tabular}{lcc}
\hline Brand & Zaldem CR & Bi-tildiem \\
\hline Adizem-XL & 67.55 & 33.34 \\
\hline Bi-tildiem & 34.13 & - \\
\hline
\end{tabular}

compared. The dissolution profiles of Adizem-XL in fasted and fed conditions (Figure 4 ) are similar, with an $f_{2}$ value of 62.18. The result suggests that the drug can be taken irrespective of the content of the Gl tract. The same holds true for Zaldem CR (Figure 5), which exhibits an $f_{2}$ value of 70.42. Bi-Tildiem dissolution profiles under fasted and fed conditions were not similar (Figure 6), with an $f_{2}$ value of 45.1 .

\section{Comparison of Apparatus 2 and 3 Dissolution Profiles}

Dissolution profiles using Apparatus 2 and $900 \mathrm{~mL}$ of SIFsp (pH 6.8) were compared with Apparatus 3 in fasted conditions. Dissolution profiles were similar for all formulations as shown in Figures 7-9. These results are confirmed by $f_{2}$ values greater than 50 (i.e., Adizem-XL, $f_{2}=69.80 ;$ Bi-Tildiem, $f_{2}=63.79 ;$ Zaldem $C R, f_{2}=64.50$ ).

\section{Kinetic Modeling of Dissolution Profiles}

As shown in Table 7, the products exhibited different drug-release mechanisms. According to dissolution

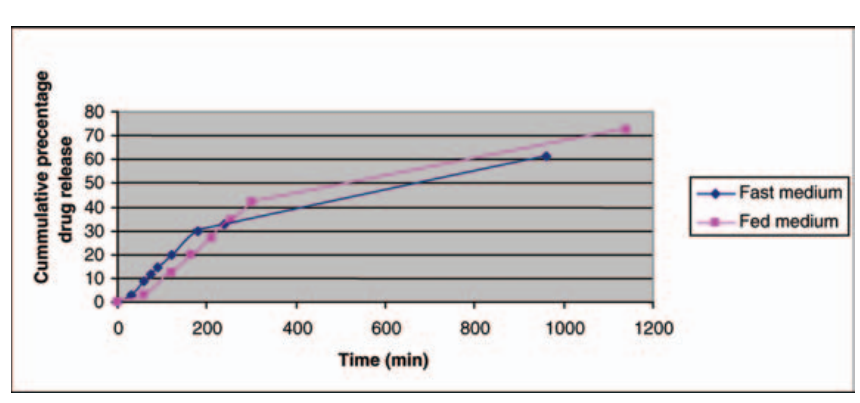

Figure 4. Dissolution profile of Adizem-XL in fasted-and fed-state conditions using USP Apparatus 3.

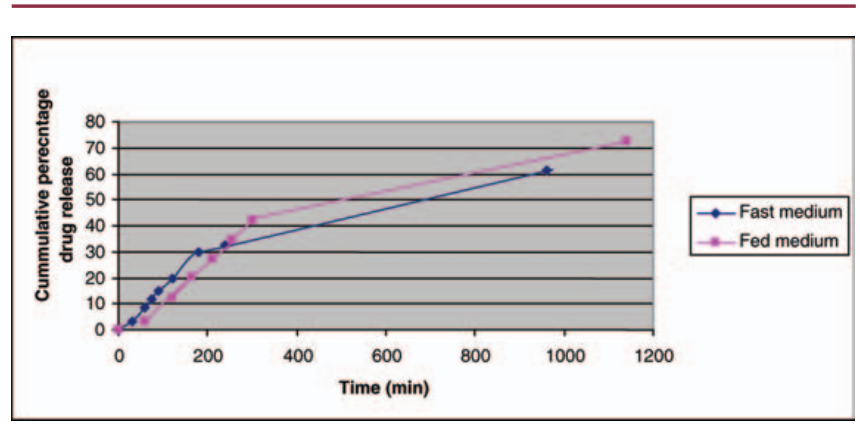

Figure 5. Dissolution profile of Zaldem CR in fasted-and fed-state conditions using USP Apparatus 3. 
Table 6. Mathematical Models Used to Describe Drug

Dissolution Curves (14)

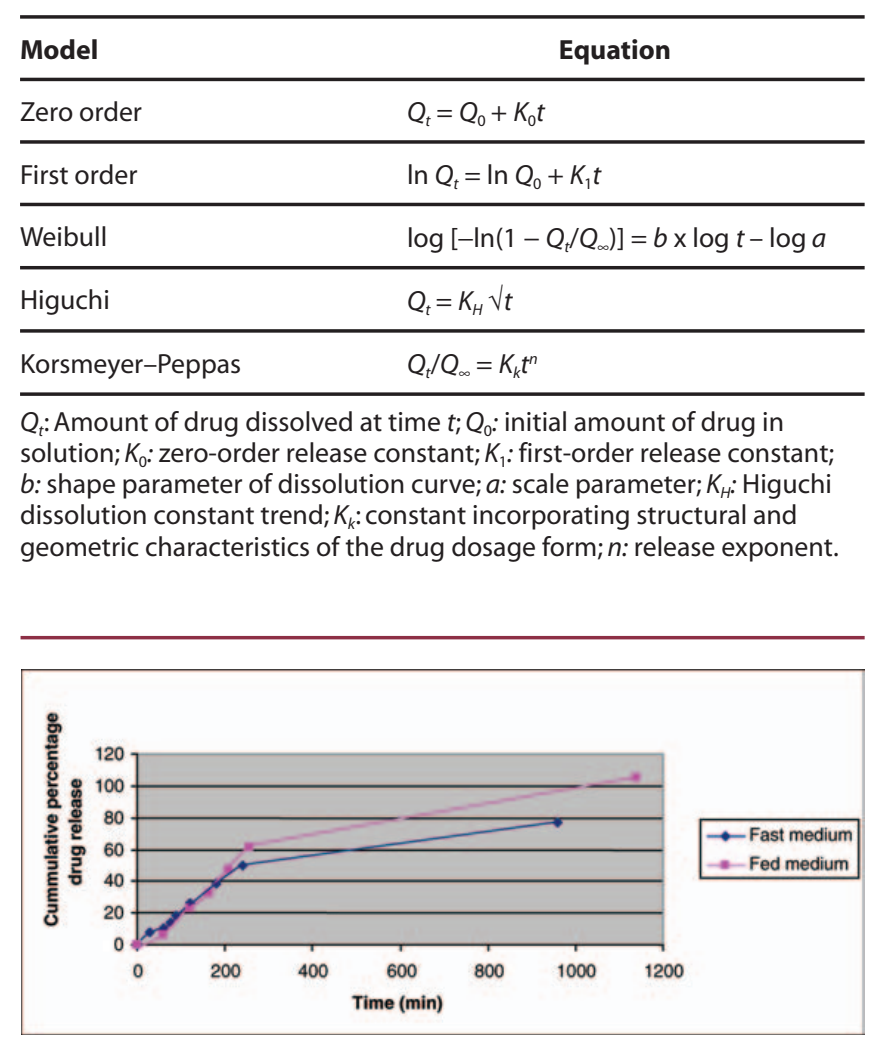

Figure 6. Dissolution profile of Bi-tildiem in fasted-and fed-state conditions using USP Apparatus 3.

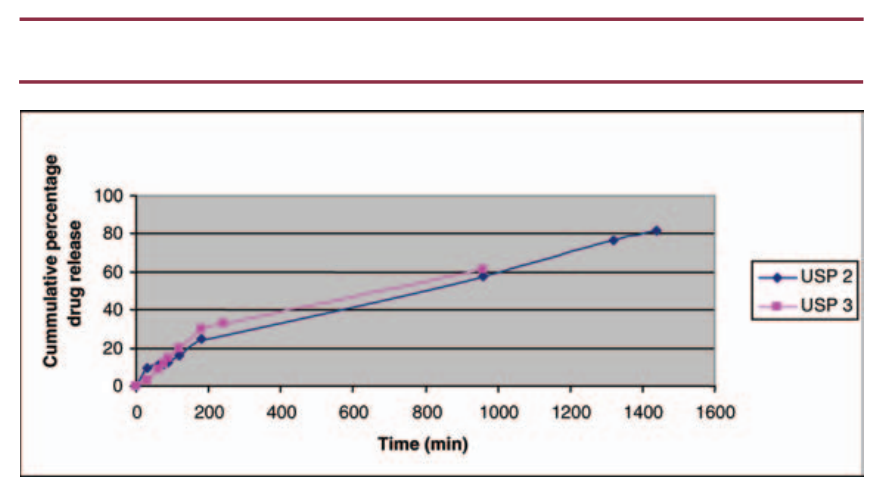

Figure 7. Dissolution profile of Adizem-XL in fasted-state conditions using USP Apparatus 2 and 3.

results from Apparatus 2, Adizem-XL tablets consisting of ethylcellulose-coated microgranules and Bi-Tildiem coated tablets exhibited zero-order drug release.

Release of drug from Zaldem tablets, which are also ethylcellulose-coated microgranules, followed the Higuchi model. The drug-release mechanism did, however, differ with the type of dissolution apparatus used. On the basis of Apparatus 3 results, dissolution profiles for all products were characterized with a zero-order drug release. Such a profile is expected according to the characteristics of the dosage forms.

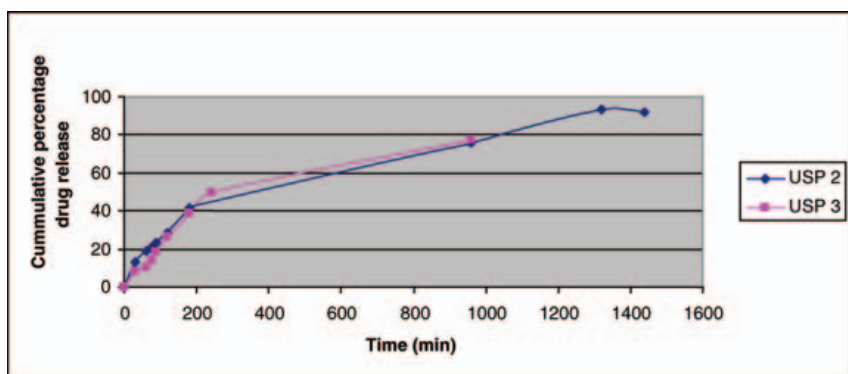

Figure 8. Dissolution profile of Bi-tildiem in fasted-state conditions using USP Apparatus 2 and 3.

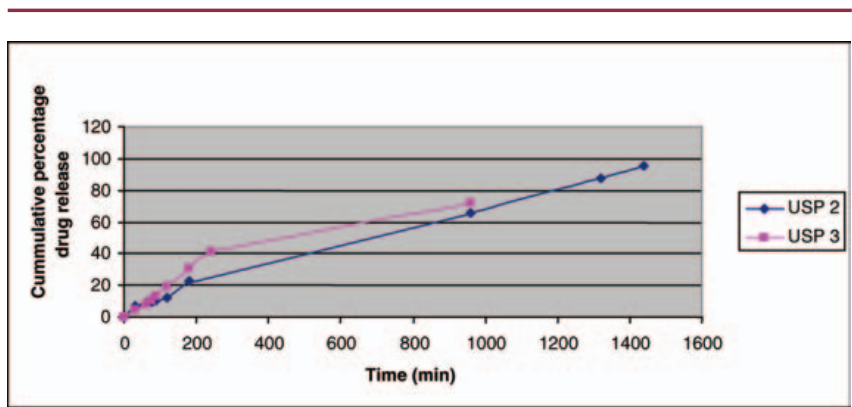

Figure 9. Dissolution profile of Zaldem CR in fasted-state conditions using USP Apparatus 2 and 3.

Table 7. Mechanism of Drug Release According to Varying Dissolution Conditions

\begin{tabular}{llcc}
\hline Product & USP 2 & USP 3 Fasted State & USP 3 Fed State \\
\hline Adizem-XL & Zero order & Zero order & Zero order \\
\hline Zaldem CR & Higuchi & Zero order & Zero order \\
\hline Bi-tildiem & Zero order & Zero order & Zero order \\
\hline
\end{tabular}

A comparison of fasted-and fed-state results using Apparatus 3 shows that dissolution behavior remained the same for a given brand. These results suggest that the drug-release mechanism is not affected by the presence of food in the Gl tract.

\section{CONCLUSION}

Although the products appeared to be similar on the basis of results generated with Apparatus 2 and 3, testing with Apparatus 3 revealed some important differences. Apparatus 3 testing provides a more discriminating and comprehensive evaluation of the release performance of ER products. Testing with Apparatus 2 and 3 under fasted conditions led to the same conclusion (i.e., the release profiles of the three products are similar). The fed conditions highlighted differences in the dissolution profile for Bi-Tildiem, suggesting a food effect on drug release. This implies that depending on the targeted 
concentration, the patient should be instructed whether to take the medication in fasted or in fed state. Zaldem and Adizem were similar in fasted or fed conditions, which suggests that the drugs can be used interchangeably. The study shows the necessity of testing products in both fasted and fed states to assess their similarity and therapeutic interchangeability. Apparatus 3 testing was more accurate in determining the release mechanism as compared with the Apparatus 2 method. No change was observed in the mechanism of drug release when comparing fasted and fed states. The USP Apparatus $3 \mathrm{pH}$-gradient method allows differentiation between individual release characteristics of diltiazem ER dosage forms. The method is a useful tool in assessing the interchangeability of ER dosage forms and in predicting food effects on the release from ER products. In vitro-in vivo correlations must be done using results obtained with Apparatus 3 to confirm these findings.

\section{REFERENCES}

1. Waiver of In Vivo Bioavailability and Bioequivalence Studies for Immediate-Release Solid Oral Dosage Forms Based on a Biopharmaceutics Classification System; Guidance for Industry; U.S. Department of Health and Human Services, Food and Drug Administration, Center for Drug Evaluation and Research (CDER), U.S. Government Printing Office:Washington, DC, August 2000. http://www.fda.gov/cder/guidance/3618fnl.pdf (accessed April 10, 2009).

2. Wu, C. Y.; Benet, L. Z. Predicting Drug Disposition via Application of BCS:Transport/Absorption/Elimination Interplay and Development of a Biopharmaceutics Drug Disposition Classification System. Pharm. Res. 2005, 22, 11-23.

3. Sweetman, S.C., Ed. Martindale: The Complete Drug Reference, 33rd ed.; Pharmaceutical Press: London, England, 2002.

4. Dressman, J.B; Reppas C. In vitro-in vivo correlation for lipophilic, poorly water-soluble drugs. Eur. J. Pharm. Sci. 2000, 11 (Supplement 2), S73-S80.

5. Galia, E.; Nicolaides, E.; Hörter, D.; Löbenberg, R.; Reppas, C.; Dressman, J.B. Evaluation of various dissolution media for predicting in vivo performance of class I and II drugs. Pharm. Res. 1998, 15, 698-705.

6. Marques, M. Dissolution media simulating fasted and fed states.Dissolution Technol. 2004, 11 (2), 16.
7. Dressman, J. B; Amidon, G. L; Reppas, C.; Shah, V. P. Dissolution testing as a prognostic tool for oral drug absorption: immediate release dosage forms. Pharm. Res. 1998, 15 (1), 11-22.

8. Kostwicz , E.S; Brauns, U.; Becker, R.; Dressman, J. B. Forecasting the oral absorption behavior of poorly soluble weak bases using solubility and dissolution studies in biorelevant media. Pharm. Res. 2002, 19 (3), 345-349.

9. Nicolaides, E.; Symillides, M.; Dressman, J. B.; Reppas, C. Biorelevant dissolution testing to predict the plasma profile of lipophilic drugs after oral administration. Pharm. Res. 2001, 18 (3), 380-388.

10. Hörter, D.; Dressman, J. B. Influence of physicochemical properties on dissolution of drugs in the gastrointestinal tract. Adv. Drug Deliver. Rev. 2001, 46, 75-87.

11. Klein, S.; Stippler, E.; Wunderlich, M.; Dressman, J. Development of dissolution tests on the basis of gastrointestinal physiology. In Pharmaceutical Dissolution Testing; Dressman, J., Kramer, J., Eds.; Taylor and Francis Group: Boca Raton, FL, 2005; pp 193-227.

12. Galia, E.; Horton, J.; Dressman, J. B. Albendazole generics - a comparative in vitro study. Pharm. Res. 1999, 16, 1871-1875.

13. Klein, S.; Butler, J.; Hempenstall, J. M.; Reppas, C.; Dressman, J.B. Media to simulate postprandial stomach. I. Matching the physicochemical characteristics of standard breakfasts. J. Pharm. Pharmacol. 2004, 56 (5), 605-610.

14. Kalantzi, L.; Goumas, K.; Kalioras, V.; Abrahamsson, B.; Dressman, J. B, Reppas, C. Characterization of the human upper gastrointestinal contents under conditions simulating bioavailability/bioequivalence studies. Pharm. Res. 2006, 23 (1), 165-176.

15. Ensure Vanilla Flavor, Package Insert. Abbott Laboratories. http://ensure.com/products/index.aspx (accessed April 10, 2009).

16. Rohrs, B. R.; Burch-Clark, D. L.; Witt, M. J.; Stelzer, D. J. USP dissolution apparatus 3 (reciprocating cylinder) instrument parameter effects on drug release from sustained release formulations. J. Pharm. Sci. 1995, 84 (8), 922-926.

17. Costa, P.; Lobo, J.M. S. Modeling and comparison of dissolution profiles. Eur.J. Pharm. Sci. 2001, 13 (2), 123-133. 